\title{
On some new sequence spaces defined by infinite matrix and modulus
}

\section{Ekrem Savaş*}

\section{"Correspondence:}

ekremsavas@yahoo.com

Department of Mathematics,

Istanbul Ticaret University, Üsküdar,

Istanbul, Turkey

\begin{abstract}
The goal of this paper is to introduce and study some properties of some sequence spaces that are defined using the $\varphi$-function and the generalized three parametric real matrix A. Also, we define A-statistical convergence.

MSC: Primary 40H05; secondary 40C05

Keywords: modulus function; almost convergence; lacunary sequence; $\varphi$-function; statistical convergence; $\mathbf{A}$-statistical convergence
\end{abstract}

\section{Introduction and background}

Let $s$ denote the set of all real and complex sequences $x=\left(x_{k}\right)$. By $l_{\infty}$ and $c$, we denote the Banach spaces of bounded and convergent sequences $x=\left(x_{k}\right)$ normed by $\|x\|=\sup _{n}\left|x_{n}\right|$, respectively. A linear functional $L$ on $l_{\infty}$ is said to be a Banach limit [1] if it has the following properties:

(1) $L(x) \geq 0$ if $n \geq 0$ (i.e., $x_{n} \geq 0$ for all $n$ ),

(2) $L(e)=1$, where $e=(1,1, \ldots)$,

(3) $L(D x)=L(x)$, where the shift operator $D$ is defined by $D\left(x_{n}\right)=\left\{x_{n+1}\right\}$.

Let $B$ be the set of all Banach limits on $l_{\infty}$. A sequence $x \in \ell_{\infty}$ is said to be almost convergent if all Banach limits of $x$ coincide. Let $\hat{c}$ denote the space of almost convergent sequences. Lorentz [2] has shown that

$$
\hat{c}=\left\{x \in l_{\infty}: \lim _{m} t_{m, n}(x) \text { exists uniformly in } n\right\},
$$

where

$$
t_{m, n}(x)=\frac{x_{n}+x_{n+1}+x_{n+2}+\cdots+x_{n+m}}{m+1} .
$$

By a lacunary $\theta=\left(k_{r}\right), r=0,1,2, \ldots$, where $k_{0}=0$, we shall mean an increasing sequence of non-negative integers with $k_{r}-k_{r-1} \rightarrow \infty$ as $r \rightarrow \infty$. The intervals determined by $\theta$ will be denoted by $I_{r}=\left(k_{r-1}, k_{r}\right]$ and $h_{r}=k_{r}-k_{r-1}$. The ratio $\frac{k_{r}}{k_{r-1}}$ will be denoted by $q_{r}$.

The space of lacunary strongly convergent sequences $N_{\theta}$ was defined by Freedman $e t a l$. [3] as follows:

$$
N_{\theta}=\left\{x=\left(x_{k}\right): \lim _{r} \frac{1}{h_{r}} \sum_{k \in I_{r}}\left|x_{k}-l e\right|=0 \text { for some } l\right\} .
$$

C 2013 Savas; licensee Springer. This is an Open Access article distributed under the terms of the Creative Commons Attribution License (http://creativecommons.org/licenses/by/2.0), which permits unrestricted use, distribution, and reproduction in any medium, provided the original work is properly cited. 
There is a strong connection between $N_{\theta}$ and the space $w$ of strongly Cesàro summable sequences which is defined by

$$
w=\left\{x=\left(x_{k}\right): \lim _{n} \frac{1}{n} \sum_{k=0}^{n}\left|x_{k}-l e\right|=0 \text { for some } l\right\} .
$$

In the special case where $\theta=\left(2^{r}\right)$, we have $N_{\theta}=w$.

More results on lacunary strong convergence can be seen from [4-11].

Ruckle [12] used the idea of a modulus function $f$ to construct a class of FK spaces

$$
L(f)=\left\{x=\left(x_{k}\right): \sum_{k=1}^{\infty} f\left(\left|x_{k}\right|\right)<\infty\right\} .
$$

The space $L(f)$ is closely related to the space $l_{1}$ which is an $L(f)$ space with $f(x)=x$ for all real $x \geq 0$.

Maddox [13] introduced and examined some properties of the sequence spaces $w_{0}(f)$, $w(f)$ and $w_{\infty}(f)$ defined using a modulus $f$, which generalized the well-known spaces $w_{0}$, $w$ and $w_{\infty}$ of strongly summable sequences.

Recently Savaş [14] generalized the concept of strong almost convergence by using a modulus $f$ and examined some properties of the corresponding new sequence spaces. Waszak [15] defined the lacunary strong $(A, \varphi)$-convergence with respect to a modulus function.

Following Ruckle, a modulus function $f$ is a function from $[0, \infty)$ to $[0, \infty)$ such that

(i) $f(x)=0$ if and only if $x=0$,

(ii) $f(x+y) \leq f(x)+f(x)$ for all $x, y \geq 0$,

(iii) $f$ increasing,

(iv) $f$ is continuous from the right at zero.

Since $|f(x)-f(y)| \leq f(|x-y|)$, it follows from condition (iv) that $f$ is continuous on $[0, \infty)$.

By a $\varphi$-function we understood a continuous non-decreasing function $\varphi(u)$ defined for $u \geq 0$ and such that $\varphi(0)=0, \varphi(u)>0$ for $u>0$ and $\varphi(u) \rightarrow \infty$ as $u \rightarrow \infty$.

A $\varphi$-function $\varphi$ is called no weaker than a $\varphi$-function $\psi$ if there are constants $c, b, k, l>0$ such that $c \psi(l u) \leq b \varphi(k u)$ (for all large $u$ ) and we write $\psi \prec \varphi$.

$\varphi$-functions $\varphi$ and $\psi$ are called equivalent and we write $\varphi \sim \psi$ if there are positive constants $b_{1}, b_{2}, c, k_{1}, k_{2}, l$ such that $b_{1} \varphi\left(k_{1} u\right) \leq c \psi(l u) \leq b_{2} \varphi\left(k_{2} u\right)$ (for all large $u$ ).

A $\varphi$-function $\varphi$ is said to satisfy $\left(\Delta_{2}\right)$-condition (for all large $u$ ) if there exists a constant $K>1$ such that $\varphi(2 u) \leq K \varphi(u)$.

In the present paper, we introduce and study some properties of the following sequence space that is defined using the $\varphi$-function and the generalized three parametric real matrix.

\section{Main results}

Let $\varphi$ and $f$ be a given $\varphi$-function and a modulus function, respectively. Moreover, let $\mathbf{A}=\left(a_{n k}(i)\right)$ be the generalized three parametric real matrix, and let a lacunary sequence $\theta$ be given. Then we define

$$
N_{\theta}^{0}(\mathbf{A}, \varphi, f)=\left\{x=\left(x_{k}\right): \lim _{r} \frac{1}{h_{r}} \sum_{n \in I_{r}} f\left(\left|\sum_{k=1}^{\infty} a_{n k}(i) \varphi\left(\left|x_{k}\right|\right)\right|\right)=0 \text { uniformly in } i\right\} .
$$


If $x \in N_{\theta}^{0}(\mathbf{A}, \varphi, f)$, the sequence $x$ is said to be lacunary strong $(\mathbf{A}, \varphi)$-convergent to zero with respect to a modulus $f$. When $\varphi(x)=x$, for all $x$, we obtain

$$
N_{\theta}^{0}(\mathbf{A}, f)=\left\{x=\left(x_{k}\right): \lim _{r} \frac{1}{h_{r}} \sum_{n \in I_{r}} f\left(\left|\sum_{k=1}^{\infty} a_{n k}(i)\left(\left|x_{k}\right|\right)\right|\right)=0 \text { uniformly in } i\right\} .
$$

If we take $f(x)=x$, we write

$$
N_{\theta}^{0}(\mathbf{A}, \varphi)=\left\{x=\left(x_{k}\right): \lim _{r} \frac{1}{h_{r}} \sum_{n \in I_{r}}\left|\sum_{k=1}^{\infty} a_{n k}(i) \varphi\left(\left|x_{k}\right|\right)\right|=0 \text { uniformly in } i\right\} .
$$

If we take $\mathbf{A}=I$ and $\varphi(x)=x$ respectively, then we have [16]

$$
N_{\theta}^{0}=\left\{x=\left(x_{k}\right): \lim _{r} \frac{1}{h_{r}} \sum_{n \in I_{r}} f\left(\left|x_{k}\right|\right)=0 \text { uniformly in } i\right\} \text {. }
$$

If we define the matrix $A=\left(a_{n k}(i)\right)$ as follows: for all $i$,

$$
a_{n k}(i):= \begin{cases}\frac{1}{n} & \text { if } n \geq k \\ 0 & \text { otherwise }\end{cases}
$$

then we have

$$
\begin{aligned}
& N_{\theta}^{0}(\mathbf{C}, \varphi, f)=\left\{x=\left(x_{k}\right): \lim _{r} \frac{1}{h_{r}} \sum_{n \in I_{r}} f\left(\left|\frac{1}{n} \sum_{k=1}^{n} \varphi\left(\left|x_{k}\right|\right)\right|\right)=0 \text { uniformly in } i\right\}, \\
& a_{n k}(i):= \begin{cases}\frac{1}{n} & \text { if } i \leq k \leq i+n-1, \\
0 & \text { otherwise, }\end{cases}
\end{aligned}
$$

then we have

$$
N_{\theta}^{0}(\hat{c}, \varphi, f)=\left\{x=\left(x_{k}\right): \lim _{r} \frac{1}{h_{r}} \sum_{n \in I_{r}} f\left(\left|\frac{1}{n} \sum_{k=i}^{i+n} \varphi\left(\left|x_{k}\right|\right)\right|\right)=0 \text { uniformly in } i\right\} .
$$

We are now ready to write the following theorem.

Theorem 2.1 Let $\mathbf{A}=\left(a_{n k}(i)\right)$ be the generalized three parametric real matrix, and let the $\varphi$-function $\varphi(u)$ satisfy the condition $\left(\Delta_{2}\right)$. Then the following conditions are true.

(a) If $x=\left(x_{k}\right) \in w(\mathbf{A}, \varphi, f)$ and $\alpha$ is an arbitrary number, then $\alpha x \in w(\mathbf{A}, \varphi, f)$.

(b) If $x, y \in w(\mathbf{A}, \varphi, f)$, where $x=\left(x_{k}\right), y=\left(y_{k}\right)$ and $\alpha, \beta$ are given numbers, then $\alpha x+\beta y \in w(\mathbf{A}, \varphi, f)$.

The proof is a routine verification by using standard techniques and hence is omitted.

Theorem 2.2 Let $f$ be any modulus function, and let the generalized three parametric real matrix $A$ and the sequence $\theta$ be given. If

$$
w(\mathbf{A}, \varphi, f)=\left\{x=\left(x_{k}\right): \lim _{m} \frac{1}{m} \sum_{n=1}^{m} f\left(\left|\sum_{k=1}^{\infty} a_{n k}(i) \varphi\left(\left|x_{k}\right|\right)\right|\right)=0 \text { uniformly in } i\right\},
$$

then the following relations are true. 
(a) If $\liminf _{r} q_{r}>1$, then we have $w(A, \varphi, f) \subseteq N_{\theta}^{0}(\mathbf{A}, \varphi, f)$.

(b) If $\sup _{r} q_{r}<\infty$, then we have $N_{\theta}^{0}(\mathbf{A}, \varphi, f) \subseteq w(A, \varphi, f)$.

(c) $1<\liminf _{r} q_{r} \leq \limsup _{r} q_{r}<\infty$, then we have $N_{\theta}^{0}(\mathbf{A}, \varphi, f)=w(\mathbf{A}, \varphi, f)$.

Proof (a) Let us suppose that $x \in w(A, \varphi, f)$. There exists $\delta>0$ such that $q_{r}>1+\delta$ for all $r \geq 1$, and we have $h_{r} / k_{r} \geq \delta /(1+\delta)$ for sufficiently large $r$. Then, for all $i$,

$$
\begin{gathered}
\frac{1}{k_{r}} \sum_{n=1}^{k_{r}} f\left(\left|\sum_{k=1}^{\infty} a_{n k}(i) \varphi\left(\left|x_{k}\right|\right)\right|\right) \\
\geq \frac{1}{k_{r}} \sum_{n \in I_{r}} f\left(\left|\sum_{k=1}^{\infty} a_{n k}(i) \varphi\left(\left|x_{k}\right|\right)\right|\right) \\
=\frac{h_{r}}{k_{r}} \frac{1}{h_{r}} \sum_{n \in I_{r}} f\left(\left|\sum_{k=1}^{\infty} a_{n k}(i) \varphi\left(\left|x_{k}\right|\right)\right|\right) \\
\geq \frac{\delta}{1+\delta} \frac{1}{h_{r}} \sum_{n \in I_{r}} f\left(\left|\sum_{k=1}^{\infty} a_{n k} \varphi\left(\left|x_{k}\right|\right)\right|\right) .
\end{gathered}
$$

Hence, $x \in N_{\theta}^{0}(\mathbf{A}, \varphi, f)$.

(b) If $\lim \sup _{r} q_{r}<\infty$, then there exists $M>0$ such that $q_{r}<M$ for all $r \geq 1$. Let $x \in$ $N_{\theta}^{0}(\mathbf{A}, \varphi, f)$ and $\varepsilon$ be an arbitrary positive number, then there exists an index $j_{0}$ such that for every $j \geq j_{0}$ and all $i$,

$$
R_{j}=\frac{1}{h_{j}} \sum_{n \in I_{r}} f\left(\left|\sum_{k=1}^{\infty} a_{n k}(i) \varphi\left(\left|x_{k}\right|\right)\right|\right)<\varepsilon
$$

Thus, we can also find $K>0$ such that $R_{j} \leq K$ for all $j=1,2, \ldots$. Now, let $m$ be any integer with $k_{r-1} \leq m \leq k_{r}$, then we obtain, for all $i$,

$$
I=\frac{1}{m} \sum_{n=1}^{m} f\left(\left|\sum_{k=1}^{\infty} a_{n k}(i) \varphi\left(\left|x_{k}\right|\right)\right|\right) \leq \frac{1}{k_{r-1}} \sum_{n=1}^{k_{r}} f\left(\left|\sum_{k=1}^{\infty} a_{n k}(i) \varphi\left(\left|x_{k}\right|\right)\right|\right)=I_{1}+I_{2},
$$

where

$$
\begin{aligned}
& I_{1}=\frac{1}{k_{r-1}} \sum_{j=1}^{j_{0}} \sum_{n \in I_{j}} f\left(\left|\sum_{k=1}^{\infty} a_{n k}(i) \varphi\left(\left|x_{k}\right|\right)\right|\right), \\
& I_{2}=\frac{1}{k_{r-1}} \sum_{j=j_{0+1}}^{m} \sum_{n \in I_{j}} f\left(\left|\sum_{k=1}^{\infty} a_{n k}(i) \varphi\left(\left|x_{k}\right|\right)\right|\right) .
\end{aligned}
$$

It is easy to see that

$$
\begin{aligned}
I_{1} & =\frac{1}{k_{r-1}} \sum_{j=1}^{j_{0}} \sum_{n \in I_{j}} f\left(\left|\sum_{k=1}^{\infty} a_{n k}(i) \varphi\left(\left|x_{k}\right|\right)\right|\right) \\
& =\frac{1}{k_{r-1}}\left(\sum_{n \in I_{1}} f\left(\left|\sum_{k=1}^{\infty} a_{n k}(i) \varphi\left(\left|x_{k}\right|\right)\right|\right)+\cdots+\sum_{n \in I_{j}} f\left(\left|\sum_{k=1}^{\infty} a_{n k}(i) \varphi\left(\left|x_{k}\right|\right)\right|\right)\right)
\end{aligned}
$$




$$
\begin{aligned}
& \leq \frac{1}{k_{r-1}}\left(h_{1} R_{1}+\cdots+h_{j_{0}} R_{j_{0}}\right) \\
& \leq \frac{1}{k_{r-1}} j_{0} k_{j_{0}} \sup _{1 \leq i \leq j_{0}} R_{i} \\
& \leq \frac{j_{0} k_{j_{0}}}{k_{r-1}} K .
\end{aligned}
$$

Moreover, we have, for all $i$,

$$
\begin{aligned}
I_{2} & =\frac{1}{k_{r-1}} \sum_{j=j_{0}+1}^{m} \sum_{n \in I_{j}} f\left(\left|\sum_{k=1}^{\infty} a_{n k} \varphi\left(\left|x_{k}\right|\right)\right|\right) \\
& =\frac{1}{k_{r-1}} \sum_{j=j_{0}+1}^{m} \frac{1}{h_{j}} \sum_{n \in I_{j}} f\left(\left|\sum_{k=1}^{\infty} a_{n k} \varphi\left(\left|x_{k}\right|\right)\right|\right) h_{j} \\
& \leq \varepsilon \frac{1}{k_{r-1}} \sum_{j=j_{0}+1}^{m} h_{j} \\
& \leq \varepsilon \frac{k_{r}}{k_{r-1}} \\
& =\varepsilon q_{r}<\varepsilon \cdot M .
\end{aligned}
$$

Thus $I \leq \frac{j_{0} k_{j_{0}}}{k_{r-1}} K+\varepsilon \cdot M$. Finally, $x \in w(A, \psi, f)$.

The proof of (c) follows from (a) and (b). This completes the proof.

We now prove the following theorem.

Theorem 2.3 Letf be a modulus function. Then $N_{\theta}^{0}(A, \varphi) \subset N_{\theta}^{0}(A, \varphi, f)$.

Proof Let $x \in N_{\theta}^{0}(A, \varphi)$. Let $\varepsilon>0$ be given and choose $0<\delta<1$ such that $f(x)<\varepsilon$ for every $x \in[0, \delta]$. We can write

$$
\frac{1}{h_{r}} \sum_{n \in I_{r}} f\left|\sum_{k=1}^{\infty} a_{n k}(i) \varphi\left(\left|x_{k}\right|\right)\right|=S_{1}+S_{2},
$$

where $S_{1}=\frac{1}{h_{r}} \sum_{n \in I_{r}} f\left(\left|\sum_{k=1}^{\infty} a_{n k}(i) \varphi\left(\left|x_{k}\right|\right)\right|\right)$, and this sum is taken over

$$
\left|\sum_{k=1}^{\infty} a_{n k}(i) \varphi\left(\left|x_{k}\right|\right)\right| \leq \delta
$$

and

$$
S_{2}=\frac{1}{h_{r}} \sum_{n \in I_{r}} f\left(\left|\sum_{k=1}^{\infty} a_{n k}(i) \varphi\left(\left|x_{k}\right|\right)\right|\right),
$$

and this sum is taken over

$$
\left|\sum_{k=1}^{\infty} a_{n k}(i) \varphi\left(\left|x_{k}\right|\right)\right|>\delta .
$$


By the definition of the modulus $f$, we have $S_{1}=\frac{1}{h_{r}} \sum_{n \in I_{r}} f(\delta)=f(\delta)<\varepsilon$ and further

$$
S_{2}=f(1) \frac{1}{\delta} \frac{1}{h_{r}} \sum_{n \in I_{r}} \sum_{k=1}^{\infty} a_{n k}(i) \varphi\left(\left|x_{k}\right|\right)
$$

Therefore we have $x \in N_{\theta}^{0}(\mathbf{A}, \varphi, f)$.

This completes the proof.

\section{A-Statistical convergence}

The idea of convergence of a real sequence was extended to statistical convergence by Fast [17] (see also Schoenberg [18]) as follows: If $\mathbb{N}$ denotes the set of natural numbers and $K \subset \mathbb{N}$, then $K(m, n)$ denotes the cardinality of the set $K \cap[m, n]$. The upper and lower natural density of the subset $K$ is defined by

$$
\bar{d}(K)=\lim _{n \rightarrow \infty} \sup \frac{K(1, n)}{n} \text { and } \underline{d}(K)=\lim _{n \rightarrow \infty} \inf \frac{K(1, n)}{n} .
$$

If $\bar{d}(K)=\underline{d}(K)$, then we say that the natural density of $K$ exists and it is denoted simply by $d(K)$. Clearly, $d(K)=\lim _{n \rightarrow \infty} \frac{K(1, n)}{n}$.

A sequence $\left(x_{k}\right)$ of real numbers is said to be statistically convergent to $L$ if for arbitrary $\varepsilon>0$, the set $K(\varepsilon)=\left\{k \in \mathbb{N}:\left|x_{k}-L\right| \geq \varepsilon\right\}$ has natural density zero. Statistical convergence turned out to be one of the most active areas of research in summability theory after the work of Fridy [19] and Šalát [20].

In another direction, a new type of convergence, called lacunary statistical convergence, was introduced in [21] as follows.

A sequence $\left(x_{k}\right)_{n \in \mathbb{N}}$ of real numbers is said to be lacunary statistically convergent to $L$ (or $S_{\theta}$-convergent to $L$ ) if for any $\varepsilon>0$,

$$
\lim _{r \rightarrow \infty} \frac{1}{h_{r}}\left|\left\{k \in I_{r}:\left|x_{k}-L\right| \geq \varepsilon\right\}\right|=0,
$$

where $|A|$ denotes the cardinality of $A \subset \mathbb{N}$. In [21] the relation between lacunary statistical convergence and statistical convergence was established among other things. Moreover, Kolk [22] defined $A$-statistical convergence by using non-negative regular summability matrix.

In this section we define $(A, \varphi)$-statistical convergence by using the generalized three parametric real matrix and the $\varphi$-function $\varphi(u)$.

Let $\theta$ be a lacunary sequence, and let $\mathbf{A}=\left(a_{n k}(i)\right)$ be the generalized three parametric real matrix; let the sequence $x=\left(x_{k}\right)$, the $\varphi$-function $\varphi(u)$ and a positive number $\varepsilon>0$ be given. We write, for all $i$,

$$
K_{\theta}^{r}((A, \varphi), \varepsilon)=\left\{n \in I_{r}: \sum_{k=1}^{\infty} a_{n k}(i) \varphi\left(\left|x_{k}\right|\right) \geq \varepsilon\right\} .
$$

The sequence $x$ is said to be $(\mathbf{A}, \varphi)$-statistically convergent to a number zero if for every $\varepsilon>0$,

$$
\lim _{r} \frac{1}{k_{r}} \mu\left(K_{\theta}^{r}((A, \varphi), \varepsilon)\right)=0 \quad \text { uniformly in } n,
$$


where $\mu\left(K_{\theta}^{r}((A, \varphi), \varepsilon)\right)$ denotes the number of elements belonging to $K_{\theta}^{r}((\mathbf{A}, \varphi), \varepsilon)$. We denote by $S_{\theta}^{0}(\mathbf{A}, \varphi)$ the set of sequences $x=\left(x_{k}\right)$ which are lacunary $(\mathbf{A}, \varphi)$-statistical convergent to zero. We write

$$
S_{\theta}^{0}(\mathbf{A}, \varphi)=\left\{x=\left(x_{k}\right): \lim _{r} \frac{1}{h_{r}} \mu\left(K_{\theta}^{r}((A, \varphi), \varepsilon)\right)=0 \text { uniformly in } i\right\} .
$$

Theorem 3.1 If $\psi \prec \varphi$, then $S_{\theta}^{0}(A, \psi) \subset S_{\theta}^{0}(A, \varphi)$.

Proof By assumption we have $\psi\left(\left|x_{k}\right|\right) \leq b \varphi\left(c\left|x_{k}\right|\right)$ and we have, for all $i$,

$$
\sum_{k=1}^{\infty} a_{n k}(i) \psi\left(\left|x_{k}\right|\right) \leq b \sum_{k=1}^{\infty} a_{n k}(i) \varphi\left(c\left|x_{k}\right|\right) \leq L \sum_{k=1}^{\infty} a_{n k}(i) \varphi\left(\left|x_{k}\right|\right)
$$

for $b, c>0$, where the constant $L$ is connected with the properties of $\varphi$. Thus, the condition $\sum_{k=1}^{\infty} a_{n k}(i) \varphi\left(\left|x_{k}\right|\right) \geq 0$ implies the condition $\sum_{k=1}^{\infty} a_{n k}(i) \varphi\left(\left|x_{k}\right|\right) \geq \varepsilon$, and finally we get

$$
\mu\left(K_{\theta}^{r}((A, \varphi), \varepsilon)\right) \subset \mu\left(K_{\theta}^{r}((A, \psi), \varepsilon)\right)
$$

and

$$
\lim _{r} \frac{1}{h_{r}} \mu\left(K_{\theta}^{r}((A, \varphi), \varepsilon)\right) \leq \lim _{r} \frac{1}{h_{r}} \mu\left(K_{\theta}^{r}((A, \psi), \varepsilon)\right) .
$$

This completes the proof.

We finally prove the following theorem.

Theorem 3.2 (a) If the matrix $A$, the sequence $\theta$ and functions $f$ and $\varphi$ are given, then

$$
N_{\theta}^{0}((A, \varphi), f) \subset S_{\theta}^{0}(A, \varphi) .
$$

(b) If the $\varphi$-function $\varphi(u)$ and the matrix $A$ are given, and if the modulus function $f$ is bounded, then

$$
S_{\theta}^{0}(A, \varphi) \subset N_{\theta}^{0}((A, \varphi), f) .
$$

(c) If the $\varphi$-function $\varphi(u)$ and the matrix $A$ are given, and if the modulus function $f$ is bounded, then

$$
S_{\theta}^{0}(A, \varphi)=N_{\theta}^{0}((A, \varphi), f) .
$$

Proof (a) Let $f$ be a modulus function, and let $\varepsilon$ be a positive number. We write the following inequalities:

$$
\begin{aligned}
& \frac{1}{h_{r}} \sum_{n \in I_{r}} f\left(\left|\sum_{k=1}^{\infty} a_{n k}(i) \varphi\left(\left|x_{k}\right|\right)\right|\right) \\
& \geq \frac{1}{h_{r}} \sum_{n \in I_{r}^{1}} f\left(\left|\sum_{k=1}^{\infty} a_{n k}(i) \varphi\left(\left|x_{k}\right|\right)\right|\right)
\end{aligned}
$$




$$
\begin{aligned}
& \geq \frac{1}{h_{r}} f(\varepsilon) \sum_{n \in I_{r}^{1}} 1 \\
& \geq \frac{1}{h_{r}} f(\varepsilon) \mu\left(K_{\theta}^{r}(A, \varphi), \varepsilon\right),
\end{aligned}
$$

where

$$
I_{r}^{1}=\left\{n \in I_{r}: \sum_{k=1}^{\infty} a_{n k}(i) \varphi\left(\left|x_{k}\right|\right) \geq \varepsilon\right\} .
$$

Finally, if $x \in N_{\theta}^{0}((A, \varphi), f)$, then $x \in S_{\theta}^{0}(A, \varphi)$.

(b) Let us suppose that $x \in S_{\theta}^{0}(A, \varphi)$. If the modulus function $f$ is a bounded function, then there exists an integer $L$ such that $f(x)<L$ for $x \geq 0$. Let us take

$$
I_{r}^{2}=\left\{n \in I_{r}: \sum_{k=1}^{\infty} a_{n k}(i) \varphi\left(\left|x_{k}\right|\right)<\varepsilon\right\} .
$$

Thus we have

$$
\begin{aligned}
\frac{1}{h_{r}} \sum_{n \in I_{r}} f\left(\left|\sum_{k=1}^{\infty} a_{n k}(i) \varphi\left(\left|x_{k}\right|\right)\right|\right) \\
\leq \frac{1}{h_{r}} \sum_{n \in I_{r}^{1}} f\left(\left|\sum_{k=1}^{\infty} a_{n k}(i) \varphi\left(\left|x_{k}\right|\right)\right|\right) \\
+\frac{1}{h_{r}} \sum_{n \in I_{r}^{2}} f\left(\left|\sum_{k=1}^{\infty} a_{n k}(i) \varphi\left(\left|x_{k}\right|\right)\right|\right) \\
\leq \frac{1}{h_{r}} M \mu\left(K_{\theta}^{r}((A, \varphi), \varepsilon)\right)+f(\varepsilon) .
\end{aligned}
$$

Taking the limit as $\varepsilon \rightarrow 0$, we obtain that $x \in N_{\theta}^{0}(A, \varphi, f)$.

The proof of (c) follows from (a) and (b).

This completes the proof.

\section{Competing interests}

The author declares that they have no competing interests.

\section{Acknowledgements}

This paper was presented during the International Conference on the Theory, Methods and Applications of Nonlinear Equations' held on the campus of Texas A\&M University-Kingsville, Kingsville, TX 78363, USA on December 17-21, 2012, and submitted for conference proceedings.

Received: 22 June 2013 Accepted: 12 August 2013 Published: 19 September 2013

\section{References}

1. Banach, S: Theorie des Operations Linearies. PWN, Warsaw (1932)

2. Lorentz, GG: A contribution to the theory of divergent sequences. Acta Math. 80, 167-190 (1948)

3. Freedman, AR, Sember, JJ, Raphel, M: Some Cesaro-type summability spaces. Proc. Lond. Math. Soc. 37, 508-520 (1978)

4. Das, G, Mishra, SK: Banach limits and lacunary strong almost convergence. J. Orissa Math. Soc. 2(2), 61-70 (1983)

5. Li, J: Lacunary statistical convergence and inclusion properties between lacunary methods. Int. J. Math. Math. Sci. 23(3), 175-180 (2000) 
6. Savaş, E: On lacunary strong $\sigma$-convergence. Indian J. Pure Appl. Math. 21(4), 359-365 (1990)

7. Savaş, E, Karakaya, V: Some new sequence spaces defined by lacunary sequences. Math. Slovaca 57(4), 393-399 (2007)

8. Savaş, E, Patterson, RF: Double $\sigma$-convergence lacunary statistical sequences. J. Comput. Anal. Appl. 11(4),610-615 (2009)

9. Savaş, E: Remark on double lacunary statistical convergence of fuzzy numbers. J. Comput. Anal. Appl. 11(1), 64-69 (2009)

10. Savaş, E, Patterson, RF: Double $\sigma$-convergence lacunary statistical sequences. J. Comput. Anal. Appl. 11(4), 610-615 (2009)

11. Savaş, E: On lacunary statistical convergent double sequences of fuzzy numbers. Appl. Math. Lett. 21, 134-141 (2008)

12. Ruckle, WH: FK Spaces in which the sequence of coordinate vectors in bounded. Can. J. Math. 25, 973-978 (1973)

13. Maddox, IJ: Sequence spaces defined by a modulus. Math. Proc. Camb. Philos. Soc. 100, 161-166 (1986)

14. Savaş, E: On some generalized sequence spaces. Indian J. Pure Appl. Math. 30(5), 459-464 (1999)

15. Waszak, A: On the strong convergence in sequence spaces. Fasc. Math. 33, 125-137 (2002)

16. Pehlivan, S, Fisher, B: On some sequence spaces. Indian J. Pure Appl. Math. 25(10), 1067-1071 (1994)

17. Fast, H: Sur la convergence statistique. Colloq. Math. 2, 241-244 (1951)

18. Schoenberg, IJ: The integrability of certain functions and related summability methods. Am. Math. Mon. 66, 361-375 (1959)

19. Fridy, JA: On statistical convergence. Analysis 5, 301-313 (1985)

20. Šalát, T: On statistically convergent sequences of real numbers. Math. Slovaca 30, 139-150 (1980)

21. Fridy, JA, Orhan, C: Lacunary statistical convergence. Pac. J. Math. 160, 43-51 (1993)

22. Kolk, E: Matrix summability of statistically convergent sequences. Analysis 13, 77-83 (1993)

doi:10.1186/1687-1847-2013-274

Cite this article as: Savaş: On some new sequence spaces defined by infinite matrix and modulus. Advances in

Difference Equations 2013 2013:274

\section{Submit your manuscript to a SpringerOpen ${ }^{\ominus}$ journal and benefit from:}

- Convenient online submission

- Rigorous peer review

- Immediate publication on acceptance

- Open access: articles freely available online

- High visibility within the field

- Retaining the copyright to your article 